\title{
RANKING EXTREME AND NON-EXTREME EFFICIENT DECISION MAKING UNITS IN DATA ENVELOPMENT ANALYSIS
}

\author{
Golamreza Jahanshahloo ${ }^{1}$, Farhad Hosseinzadeh Lotfi ${ }^{1}$, Naghi Shoja ${ }^{2}$, Mehdi Fallah \\ Jelodar $^{1^{*}}$ Amir Gholam Abri ${ }^{1}$ \\ ${ }^{1}$ Department of Mathematics, Science and Research Branch,Islamic Azad \\ University,Tehran,Iran. \\ ${ }^{2}$ Department of Mathematics, Firoozkooh branch, Islamic Azad University, Firoozkooh, \\ Iran. \\ mehdi.fallah_jelodar@yahoo.com
}

\begin{abstract}
In evaluating decision making units (DMU) by using Data Envelopment Analysis (DEA) technique, it happens that more than one unit got efficiency score one. In such a case there should be some criterion for ranking these DMUs. Up to now, all of DEA model could rank only extreme efficient units. In this paper the authors proposed a method for ranking extreme and non extreme efficient units.
\end{abstract}

Key Words: Data Envelopment Analysis (DEA), Efficiency, Ranking.

\section{INTRODUCTION}

Data Envelopment Analysis (DEA) is a non parametric method for evaluating decision making units (DMU). It has been proposed by Charnes et al [8], in assessment of an educational center in USA and extended by Banker et al [5]. They used mathematical programming to reach this aim.

One of the main objectives of DEA is to measure the efficiency of Decision Making Units (DMUs) such as schools, public agencies, banks and etc. One of the ways for determining efficiency score of DMUs is to apply the Charnes et.al [8] model (CCR model) that deals with a ratio of multiple outputs and multiple inputs. One of the most interesting research subjects in DEA is to discriminate efficient units. In section 2 we summarized some ranking methods.

The objective of this work is to propose a method for ranking all efficient units, either extreme efficient or non extreme efficient DMUs. In section three the method is illustrated and in section four an example is used to explain our purpose. Our proposed method is based on using a ranking method; for example AP model which is proposed by Anderson and Peterson [4] or other ranking models; and finding strong efficient hyperplane which define production possibility set (PPS).

In evaluating the relative efficiency of each Decision making units (DMUs) by DEA models, we obtain efficiency scores between zero and one. In this way, usually more than one unit may be efficient in DEA models and their efficiency scores are 1. Also, pay attention that the number of efficient units in the Variable return to scale (VRS) models is not less than the Constant return to scale (CRS) models. Therefore, the researchers proposed some methods to discriminant these efficient units. This concept has named Ranking efficient units in DEA. There are lots of ranking methods and each of them has special quality and property to rank efficient units. 
Charnes et all [6], counted the number of times that an efficient DMU play the role of benchmark unit for others, and used this norm to rank these units. Since, finding the reference set of a DMU is not easy, their model is not a suitable method. Charnes et all [7], proposed another method to find the benchmark DMUs. They changed the rate of outputs and evaluate the change of efficiency score. However, they didn't distinguish how they can do it.

Sexton et all [17], suggested the cross efficiency method. In this model they used the weights which they had obtained them by solving each of n-linear problems. They evaluate the efficiency of each DMU, $\mathrm{n}$ times and store these data in a matrix. Each row of this matrix contains the cross efficiency score of DMUs. They computed the average of these rows and store the result as a ranking measure. It seams this is an acceptable method, but it may has some difficulties. The biggest problem appears when the DEA models have alternative solutions.

Finally, it should be noted that there are some techniques and strategies in DEA which they affect on ranking. For example, Thompson et al [18], used the assurance regions. In their technique, the number of efficient DMUs may be decrease. But it isn't a suitable method because finding suitable weights isn't easy. Adler et al [2], proposed another method to difference between DMUs. In their model they decrease the number of inputs and outputs by component analysis. Therefore, the number of efficient DMUs is decreased. But in general this model couldn't use for a complete ranking.

Anderson and Petersen (AP model) [4], have ranked extreme efficient units by omitting them from Possibility Production Set (PPS), and then Mehrabian et al (MAJ) [15], have modified the AP model. In some circumstances, the mentioned models may be infeasible and specially the AP model may be unstable because of extreme sensitivity to small variations in data, where some DMUs have relatively small values for some of the inputs. Saati et al [16], have modified MAJ model and solved its infeasibility and Jahanshahloo et al [13], have changed the type of data normalization in order to receive a much better result. In order to remove the difficulties from AP and MAJ models, some mathematicians have used specific norms. For instance, Jahanshahloo et al [10], have practiced $L$, norm for ranking efficient units. Amirteimoori et al [3], have experienced $L$, norm to find the gap between evaluated efficient units and the new PPS. Gradient line and ellipsoid norms have been used by Jahanshahloo et al [14], in order to rank efficient units. Tone [19] and [20], has used SBM model in this way. To review ranking methods see also Adler et al [1].

This paper is organized as follows: section 1 is introduction. Record of ranking will coming in section. We presented our method in section 3 and illustrated a numerical example in section 4. Finally, section 5 is conclusion.

\section{DEA BACKGROUND}

In this section we are going to summarize some DEA and ranking models. In this way consider $n, D M U s$ with $m$ inputs and $s$ outputs. The input and output vectors of $\quad D_{j} \quad(j=1, \ldots, n) \quad$ are $\quad X_{j}=\left(x_{1 j}, \ldots, x_{m j}\right)^{t}, Y_{j}=\left(y_{1 j}, \ldots, y_{s j}\right)^{t} \quad$ where
$X_{j} \geq 0, X_{j} \neq 0, Y_{j} \geq 0, Y_{j} \neq 0$.

By using the variable return to scale, convexity and possibility postulates, the 
non-empty production possibility set (PPS) is defined as follows:

$$
T_{v}=\left\{(X, Y): X \geq \sum_{j=1}^{n} \lambda_{j} X_{j}, Y \leq \sum_{j=1}^{n} \lambda_{j} Y_{j}, \sum_{j=1}^{n} \lambda_{j}=1, \lambda_{j} \geq 0, j=1 \ldots, n\right\}
$$

By the above definition the BCC model proposed by Banker et al [7] is as follows:

$$
\begin{array}{cl}
\min \quad \theta-\varepsilon\left[\sum_{i=1}^{m} s_{i}^{-}+\sum_{r=1}^{s} s_{r}^{+}\right] \\
\text {S.t } \quad \sum_{j=1}^{n} \lambda_{j} x_{i j}+s_{i}^{-}=\theta x_{i p}, \quad i=1, \ldots, m \\
\sum_{j=1}^{n} \lambda_{j} y_{r j}-s_{r}^{+}=y_{r p}, \quad r=1, \ldots, s \\
\sum_{j=1}^{n} \lambda_{j}=1 \\
\lambda_{j} \geq 0, \quad j=1, \ldots, n \\
s_{i}^{-} \geq 0, \quad i=1, \ldots, m \\
s_{r}^{+} \geq 0, \quad r=1, \ldots, s
\end{array}
$$

and its dual is:

$$
\begin{aligned}
\max & \sum_{r=1}^{s} u_{r} y_{r p}-u_{0} \\
\text { S.t } \quad & \sum_{i=1}^{m} v_{i} x_{i p}=1 \\
& \sum_{r=1}^{s} u_{r} y_{r j}-\sum_{i=1}^{m} v_{i} x_{i j}-u_{0} \leq 0, \quad j=1, \ldots, n \\
& u_{r} \geq \varepsilon, \quad r=1, \ldots, s \\
& v_{i} \geq \varepsilon, \quad i=1 \ldots, m
\end{aligned}
$$

Clearly, the evaluated $D M U_{p}$ is efficient if and only if $\theta^{*}=1$ and all slack variables in every optimal solution be zero in problem (1). Equivalently $D M U_{p}$ is efficient if and only if there exists an optimal solution for problem (2) such that $\left(U^{*}, V^{*}\right)>0 \quad$ and $\quad \sum_{r=1}^{s} u_{r} y_{r p}-u_{0}=1 \quad$. Then the hyperplane $H=\left\{(X, Y): U^{*} Y-V^{*} X-u_{0}=0\right\}$ is said to be strong efficient hyperplane when there exists an optimal solution $\left(U^{*}, V^{*}, u_{0}^{*}\right)$ of problem (2) which $U^{*}>0$ and $V^{*}>0$ and $U^{*} Y-u_{0}^{*}=1$.

Anderson and Peterson [4] by omission of efficient units have been ranked these DMUs. Their proposed model is as follows: 


$$
\begin{aligned}
\max \quad \sum_{r=1}^{s} u_{r} y_{r p}-u_{0} \\
\text { S.t } \quad \sum_{i=1}^{m} v_{i} x_{i p}=1 \\
\sum_{r=1}^{s} u_{r} y_{r j}-\sum_{i=1}^{m} v_{i} x_{i j}-u_{0} \leq 0, \quad j=1, \ldots, n, j \neq p \\
u_{r} \geq \varepsilon, \quad r=1, \ldots, s \\
v_{i} \geq \varepsilon, \quad i=1 \ldots, m
\end{aligned}
$$

Clearly, the optimal objective value of AP model is grater than 1 for extreme efficient units and equal 1 for non extreme efficient units. Therefore, AP model does not have any suggestion for ranking non extreme efficient units, and it is the problem of all DEA modes.

\section{PROPOSED METHOD FOR RANKING ALL EFFICIENT UNITS}

According to the last section, ranking efficient units has been developed because of existence numbers of efficient units. One of the most important point in ranking models is that there are no DEA models for ranking non extreme efficient DMUs. Therefore, there are no difference between these units. For example by using super efficiency models such as AP and MAJ models, efficiency score 1 is obtained again for these units. In this article we are going to construct a method for ranking all efficient units either extreme or non extreme efficient units by using DEA methods. Before explaining this method it is important to say that Jahanshahloo et.al [12] used mont carlo method for ranking all efficient units. But it is not a DEA method.

This new method is based on AP model and omission of efficient units from production possibility set (Using AP model is arbitrary and one may use other ranking models, especially when AP model occurs infeasibility). Also, strong efficient hyperplane which are binding on extreme efficient units play an important role in this method. To gain this aim the following computational steps should be done:

- Use AP model for evaluating all units and suppose it is feasible for all DMUs. Three sets of units may be considered:

$$
\begin{aligned}
& S E=\left\{D M U_{j}=\left(x_{j}, y_{j}\right): \theta_{j}^{*}>1\right\} \\
& E^{\prime}=\left\{D M U_{j}=\left(x_{j}, y_{j}\right): \theta_{j}^{*}=1\right\} \\
& F=\left\{D M U_{j}=\left(x_{j}, y_{j}\right): 0<\theta_{j}^{*}<1\right\} \\
& \theta_{j}^{*} \text { is the optimal objective value obtained by AP model in evaluating } D M U_{j} .
\end{aligned}
$$

By above definition SE is the set of extreme efficient units, $E^{\prime}$ is the set of non extreme 
efficient units and finally, $\mathrm{F}$ is the set of inefficient DMUs. Suppose that $E^{\prime} \neq \varnothing$ to continue.

- Find all strong efficient hyperplane which defines PPS and are binding on members of SE. Then construct a subset of SE which exactly $\mathrm{m}+\mathrm{s}$ strong efficient hyperplane are binding on them and name the new set by $\overline{S E}$. Rearrange members of $\overline{S E}$ by their descending ranks. Suppose $\overline{S E}$ be in following format:

$\bar{E}=\left\{D M U_{i_{1}}, \ldots, D M U_{i_{k}}\right\}$, where $D M U_{i_{1}}$ has the best rank in this set. May be there is no such DMU which $\mathrm{m}+\mathrm{s}$ strong efficient hyperplane are binding on it. In this case order members of SE by their ranks and continue the process.

At the end of this step note that for finding strong efficient hyperplane the method which is proposed by Huang and Rousseau [9], may be used and it is as follows:

$\min u_{0}$

$$
\begin{aligned}
S . t & \sum_{r=1}^{s} u_{r} y_{r p}-\sum_{i=1}^{m} v_{i} x_{i p}=u_{0}, \\
& \sum_{r=1}^{s} u_{r} y_{r j}-\sum_{i=1}^{m} v_{i} x_{i j} \leq u_{0}, \quad j=1, \ldots, n \\
& \sum_{i=1}^{m} v_{i}+\sum_{r=1}^{s} u_{r}=1 \\
& u_{r} \geq \varepsilon, \quad r=1, \ldots, s \\
& v_{i} \geq \varepsilon, \quad i=1 \ldots, m
\end{aligned}
$$

where $D M U_{p}=\left(X_{p}, Y_{p}\right) \in S E$.

- Consider $D M U_{i_{1}}$. Define a subset of $E^{\prime}$ which its members are binding on strong efficient hyperplane of $D M U_{i_{1}}$ and omit it from E'. Define $D_{1}=E^{\prime}-E_{1}{ }^{\prime}$ where $E^{\prime}$ is the set of binding hyperplane on $D M U_{i_{1}}$ and $\mathrm{D}$ is the set of the hyperplanes which are not binding on $D M U_{i_{1}}$. Then consider a subset of $D_{1}$ such that its members are binding on strong efficient hyperplane of $D M U_{i_{2}}$ and name it $E_{2}{ }^{\prime}$ and then define $D_{2}=D_{1}-E_{2}{ }^{\prime}$. Continue this process while for some $\mathrm{k}, D_{k}=\varnothing$. After above partitioning go to 4 .

- Omit $D M U_{i_{1}}$ from SE and name the remaining DMUs by $S E_{i_{1}}$. Add one of the members of $E_{1}{ }^{\prime}$ to $S E_{i_{1}}$ and use AP model for evaluating this non extreme efficient unit under new data set and store its super efficiency score. Do this process for other members of $E_{1}{ }^{\prime}$ and store their scores. Then omit $D M U_{i_{2}}$ from SE and name remaining DMUs by $S E_{i_{2}}$. Add one of the members of $E_{2}^{\prime}$ to $S E_{i_{2}}$ and use AP model for 
evaluating this unit in new data set and store its super efficiency score and do the above for other members of $E_{2}{ }^{\prime}$. Continue these process for other members of $\overline{S E}$ and store all super efficiency scores.

- Rank all efficient units by using super efficiency scores of extreme efficient units obtained by step 1 and super efficiency scores of non extreme efficient obtained by the last step.

By using the above procedure all efficient DMUs can be ranked.

\section{EXAMPLE}

In this section we constructed 3 examples to use our proposed method for ranking all efficient units. In the first example AP model is used for our proposal an i has one input and one output. $L_{1}$ norm method is used in second example because of infeasibility of AP model. The third example consists of 2 inputs and 2outputs.

\subsection{Example1:Ap Model Is Feasible}

Data are summarized in table 1 and are shown in figure 1:

Table 1: Data

\begin{tabular}{|c|c|c|c|c|c|c|c|c|c|c|c|c|c|}
\hline DMUs & A & B & C & D & E & F & G & H & I & J & K & L & M \\
\hline Input & 1 & 1.5 & 2 & 3 & 4 & 5 & 6 & 7 & 8 & 1 & 3 & 5 & 8 \\
\hline Output & 2 & 3 & 4 & 5 & 6 & 7 & 7.5 & 8 & 8 & 1 & 2 & 4 & 7 \\
\hline
\end{tabular}

The results of ranking by AP model are shown in table 2:

Table 2: The results of ranking

\begin{tabular}{|c|c|c|c|c|c|c|c|c|}
\hline $\begin{array}{c}\text { Eff } \\
\text { Units }\end{array}$ & A & B & C & D & E & F & G & H \\
\hline Score & 1.25 & 1 & 1.125 & 1 & 1 & 1.0666 & 1 & 1.1428 \\
\hline Rank & 1 & - & 3 & - & - & 4 & - & 2 \\
\hline
\end{tabular}

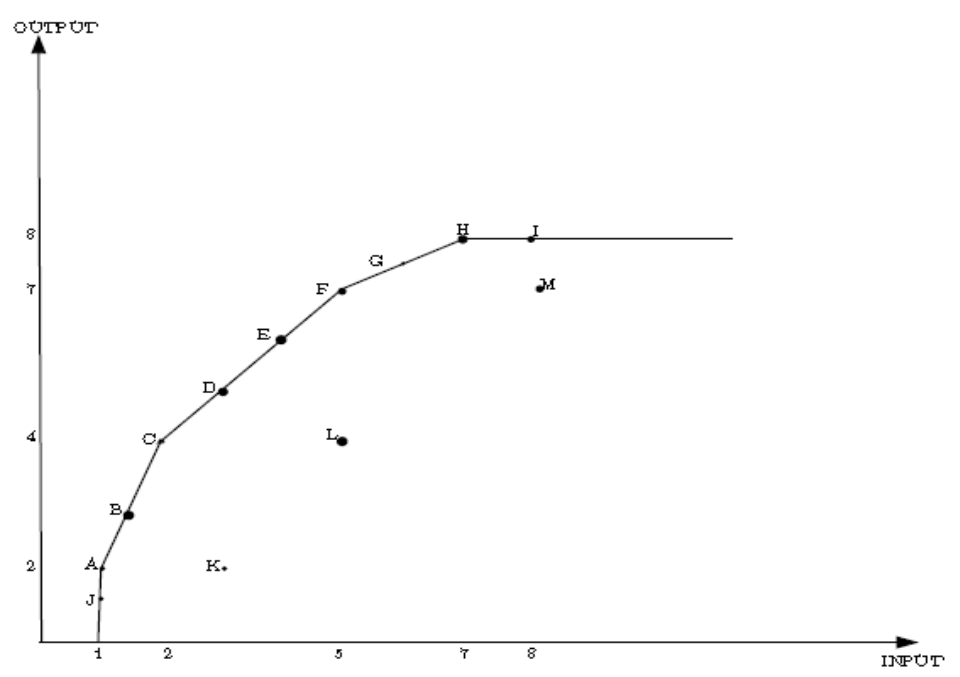

Fig 1: Data set in BCC model 
In this example $S E=\{A, C, F, H\} \quad$ and $\quad S E^{\prime}=\{B, D, E, G\} \quad$ and $F=\{I, J, K, L, M\}$. Then the set of extreme efficient units which exactly 2 strong efficient hyperplane are binding on them is: $\overline{S E}=\{C, F\}$, where the members of $\overline{S E}$ are ordered by their rank. After that it is evident from figure 1 that $E_{1}{ }^{\prime}=\{B, D, E\}$ and $E_{2}{ }^{\prime}=\{G\}$ where the members of $E_{1}{ }^{\prime}$ are the set of all non extreme efficient units which are binding on strong efficient hyperplane of unit $\mathrm{B}$ and the member of $E_{2}{ }^{\prime}$ is the set of non extreme efficient units which are binding on strong efficient hyperplane of unit $\mathrm{F}$ and are not in $E_{1}{ }^{\prime}$. Then omit DMU C from SE and name the remaining DMUs by $S E_{C}$. Add unit B to $S E_{C}$ and use AP model for evaluating this unit under new data set. Again add unit D and $\mathrm{E}$ to $S E_{C}$ separately and use AP model for evaluating them. At the end omit unit $\mathrm{f}$ from $\mathrm{SE}$ and name the remaining units by $S E_{F}$. Add unit G to $S E_{F}$ and use AP model for this unit under new data set. The super efficiency scores for these units are; $\theta_{B}^{*}=1.1111, \theta_{D}^{*}=1.0555, \theta_{E}^{*}=1.050, \theta_{G}^{*}=1.041$. The results of new ranking are summarized in table 3 :

Table 3: Results of ranking obtained by proposed model

\begin{tabular}{|c|c|c|c|c|c|c|c|c|}
\hline DMUs & A & B & C & D & E & F & G & H \\
\hline $\begin{array}{c}\text { New } \\
\text { ranking }\end{array}$ & 1 & 4 & 3 & 6 & 7 & 5 & 8 & 2 \\
\hline
\end{tabular}

Follow to the last table non extreme efficient unit B has better rank than extreme efficient unit F.

\subsection{Example2: Ap Model Is Infeasible}

Consider the following data set. It is evident that by using AP model that AP model is ifeasible for DMU H. Therefore, we use $L_{1}$ norm [10], and it is as ollows:

$$
\begin{aligned}
& \min \quad \Gamma_{c}^{o}(X, Y)=\sum_{i=1}^{m} x_{i}-\sum_{r=1}^{s} y_{r}+\alpha \\
& \text { S.t } \sum_{j=1, j \neq o}^{n} \lambda_{j} x_{i j} \leq x_{i}, i=1, \ldots, m \\
& \sum_{j=1, j \neq o} \lambda_{j} y_{r j} \geq y_{r}, r=1, \ldots, s, \\
& x_{i} \geq x_{i o}, i=1, \ldots, m \\
& y_{r} \leq y_{r o}, r=1, \ldots, s \\
& \lambda_{j} \geq 0, \quad j=1, \ldots, n, j \neq o
\end{aligned}
$$

where $\alpha=\sum_{r=1}^{s} y_{r o}-\sum_{i=1}^{m} x_{i o}$ is a constant value. The table of data and result of 
ranking is as follows:

Table 4: Data

\begin{tabular}{|c|c|c|c|c|c|c|c|c|c|c|c|c|}
\hline DMUs & $\mathrm{A}$ & $\mathrm{B}$ & $\mathrm{C}$ & $\mathrm{D}$ & $\mathrm{E}$ & $\mathrm{F}$ & $\mathrm{G}$ & $\mathrm{H}$ & $\mathrm{J}$ & $\mathrm{K}$ & $\mathrm{L}$ & $\mathrm{M}$ \\
\hline Input & 1 & 1.5 & 2 & 3 & 4 & 5 & 6 & 7 & 1 & 3 & 5 & 8 \\
\hline Output & 2 & 3 & 4 & 5 & 6 & 7 & 7.5 & 8 & 1 & 2 & 4 & 7 \\
\hline$L_{1}$ score & 0.7142 & 0 & 0.03571 & 0 & 0 & 0.03125 & 0 & 0.0625 & 0 & 0 & 0 & 0 \\
\hline
\end{tabular}

clearly, DMUB, DMUD, DMUE and DMUG are non-extreme efficient units.

By using the mentioned method the following scores are obtained: $\theta_{B}^{*}=0.04228$, $\theta_{D}^{*}=0.05714, \theta_{E}^{*}=0.02851$ and $\theta_{G}^{*}=0.03750$. By these new scores the rank of extreme efficient units are as follows:

Table 5: The result of ranking

\begin{tabular}{|c|c|c|c|c|c|c|c|c|}
\hline DMUs & A & B & C & D & E & F & G & H \\
\hline$L_{1}$ rank & 1 & 4 & 6 & 3 & 8 & 7 & 5 & 2 \\
\hline
\end{tabular}

\subsection{Example3: Multiple Inputs And Outputs}

This example consists of 2 inputs and 2 outputs. Table of data and the result of ranking are shown in the following table:

Table 6: Data

\begin{tabular}{|c|c|c|c|c|c|c|}
\hline DMUs & Input1 & Input2 & Output1 & Output2 & AP score & Ranking \\
\hline 1 & 586 & 581 & 46928 & 0.6514 & 1.001328 & 6 \\
\hline 2 & 475 & 558 & 42879 & 0.5529 & 1.015965 & 4 \\
\hline 3 & 201 & 600 & 43576 & 1.3500 & 1.848087 & 1 \\
\hline 4 & 299 & 609 & 45673 & 0.7290 & 0.988677 & - \\
\hline 5 & 318 & 613 & 40990 & 0.3190 & 0.926382 & - \\
\hline 6 & 265 & 558 & 39079 & 0.5150 & 1.019981 & 3 \\
\hline 7 & 467 & 580 & 38455 & 0.3184 & 0.941097 & - \\
\hline 8 & 583 & 625 & 54291 & 1.7158 & 1.012007 & 5 \\
\hline 9 & 347 & 535 & 34514 & 0.4512 & 1.042991 & 2 \\
\hline 10 & 296 & 650 & 41984 & 1.2195 & 0.908321 & - \\
\hline 11 & 600 & 740 & 43249 & 0.9205 & 0.772496 & - \\
\hline 12 & 575 & 775 & 43291 & 0.5825 & 0.732652 & - \\
\hline 13 & 351 & 888 & 46444 & 1.0400 & 0.863951 & - \\
\hline 14 & 283 & 727 & 41841 & 0.3210 & 0.802734 & - \\
\hline 15 & 431 & 695 & 40221 & 0.2365 & 0.801175 & - \\
\hline 16 & 590 & 626 & 54291 & 1.7158 & 0.998403 & - \\
\hline 17 & 583 & 625 & 54291 & 1.7150 & 1.000000 & Non \\
\hline & & & & & & \\
\hline
\end{tabular}

By using model 4 the efficient hyperplanes which are binding on efficient units is shown in the following table. Note that these hyperplanes are in the following format:

$$
H=\left\{(X, Y): U^{t} Y-V^{t} X=u_{0}\right\} \text { where } \mathrm{U} \text { and } \mathrm{V} \text { are the output and input weights }
$$

vectors obtained by model 4 . 
Table 7: Coefficients of defining hyperplanes

\begin{tabular}{|c|c|c|c|c|c|}
\hline No & $u_{o}$ & $V_{1}$ & $V_{2}$ & $U_{1}$ & $U_{2}$ \\
\hline 1 & $9.135734636 \mathrm{E}+1$ & 0.00001000 & 0.28946661 & 0.00162732 & 0.70889607 \\
\hline 2 & $1.227411668 \mathrm{E}+1$ & 0.00001000 & 0.02796313 & 0.00006522 & 0.97196165 \\
\hline 3 & $1.3059359742 \mathrm{E}+3$ & 0.96555695 & 0.00001000 & 0.03442305 & 0.00001000 \\
\hline 4 & $1.548190233 \mathrm{E}+1$ & 0.00244179 & 0.03625191 & 0.00012535 & 0.96118094 \\
\hline 5 & $5.4289359207 \mathrm{E}+4$ & 0.00001000 & 0.00001000 & 0.99997000 & 0.00001000 \\
\hline 6 & 7.563800029406 & 0.00021017 & 0.01547687 & 0.00001000 & 0.98430295 \\
\hline
\end{tabular}

Clearly, DMU17 is a non-extreme efficient unite and it is binding on hyperplane no. $3,4,5$ and 6 . Note that there is no extreme efficient unite which it is binding on exactly $m+s$ efficient hyperplane. Therefore, we do the proposed model for DMU3, because it has the best rank in this example. By doing this the new score of DMU17 is: 1.012000 .

\section{CONCLUSION}

In this paper we proposed a super efficiency method for ranking extreme and non extreme efficient units. AP model and the proposed model by Huang and Rousseau are used for ranking all efficient units. It is very important to note that in this method discriminant of efficient units is considered. For example unit B is a non extreme efficient units which its super efficiency score obtained by AP model is again 1. Follow to figure 1, unit $\mathrm{B}$ is better than extreme efficient units $\mathrm{F}$ and $\mathrm{H}$; and it is not considered in AP model. But discriminant of unit $B$ with units $F$ and $G$ is considered in our proposed model. Finally, note that this method may be threatened when non extreme efficient unit is very similar to extreme efficient units. For feature views the authors can remove this weakness and develop it for other ranking models.

\section{REFERENCES}

1. Adler, N., Friedman, L., Sinuany-Stern, Z., 2002. Review of ranking methods in the data envelopment analysis context. European jurnal of operational research 140, 249265.

2. Adler, N., Golany, B., 2001. Evaluation of deregulated airline networks using data envelopment analysis combined with principle component analysis with an application in Western Europe. European Journal of Operation Research 132(2), 18-31.

3. Amirteimoori, A., Jahanshahloo, G.R., Kordrostami, S., 2005. Ranking of decision making units in data envelopment analysis: A distance-based approach.Applied mathematics and computation 171, 122-135.

4. Anderson, P., Petersen, N.C., 1993. A pdrocedure for ranking efficient units in data envelopment analysis. Management science 39 (10), 1261-1264.

5. Banker, R.D., Charnes, A., Cooper, W.W., 1984. Some models for estimating technical and scale inefficiencies in data envelopment analysis. Management Science 30 (9), 1078-1092.

6. Charnes, A., Clark, C.T., Cooper, W.W., Golany, B., 1985a. A development study of data envelopment analysis in measuring the efficiency of maintenance units in US air forces. Annals of Operation Research 2, 95-112. 
7. Charnes, A., Cooper, W.W., Golany, B., Seiford, L, Stutz, J.,1985b. Foundation of data envelopment analysis for Pareto-Koopmans efficient empirical production functions. Journal of Econometrics 30, 91-107.

8. Charnes, A., Cooper, W.W., Rhodes, E., 1978. Measuring the efficiency of decision making units. European Journal of Operation Research 2, 429-444.

9. Huang Z., Rousseau J., 1997. Determining rates of changes in ata envelopment analysis. Opl Res Soc 591-599.

10. Jahanshahloo, G.R, Hosseinzadeh Lotfi, F, Shoja, N, Tohidi, G, Razavian, s, 2004. Ranking using $L_{1}$ norm in data envelopment analysis. Applied mathematics and computational 153 (1), 215-224.

11. Jahanshahloo, G.R, Hosseinzadeh Lotfi, F, Rezai Balf, F, Zhiani Rezai, H, Akbarian, D., 2004. Ranking efficient DMUs using tchebycheff norm. Working Paper.

12. Jahanshahloo, G.R, Hosseinzadeh Lotfi, F, Rezai Balf, F, Zhiani Rezai, H, 2005. Using mont carlo method for ranking efficient units.

13. Jahanshahloo, G.R., Pourkarimi, L., Zarepisheh, M., 2006. odified MAJ model for ranking decision making units in data envelopmet analysis. Applied mathematics and computation 174, 1054-1059.

14. Jahanshahloo, G.R., Sanei, M, Hosseinzadeh Lotfi, F, Shoja, N, 2004. Using the gradient line for ranking DMUs in DEA. Applied mathematics and computation 151(1), 209-219.

15. Mehrabian, S, Alirezaee, M.R, Jahanshahloo, G.R, 1999. A complete efficiency ranking of decision making units in data envelopment analysis. Computational optimization and applications 4, 261-266.

16. Saati, M.S, Zarafat Angiz, M, Jahanshahloo, G.R, 1999. A model for ranking decision making units in data envelopment analysis. Recrca operativa vol.31.

17. Sexton, T.R., Silkman, R.H., Hogan, A.J., 1986. Data envelopment analysis: Critique and extensions in: Silkman, R.H., (Ed), Measuring efficiency: An Assessment of Data envelopment analysis. Jossy-Bath, San Francisco, CA, pp 73-105.

18. Thompson, R.G,. Lee, E., Thrall, R.M., 1992. DEA/AR efficiency of US independent oil/gas producers over time. Computers and Operation Research 19(5), 377-391.

19. Tone, K, 2001. A slack-based measure of efficiency in data envelopment Analysis. European journal of operational research 130, 498-509.

20. Tone, K, 2002 A slack-based measure of efficiency in data envelopment Analysis. European journal of operational research 143, 32-41. 